This is the final peer-reviewed accepted manuscript of:

Tommaso De Cola, Daniele Tarchi, Alessandro Vanelli-Coralli

"Future trends in broadband satellite communications: information centric networks and enabling technologies"

in: INTERNATIONAL JOURNAL OF SATELLITE COMMUNICATIONS AND NETWORKING Volume 33, Issue 5, Page 473-490, Special Issue SI

The final published version is available online at: https://doi.org/10.1002/sat.1101

Rights / License:

The terms and conditions for the reuse of this version of the manuscript are specified in the publishing policy. For all terms of use and more information see the publisher's website.

This item was downloaded from IRIS Università di Bologna (https://cris.unibo.it/)

When citing, please refer to the published version. 


\title{
Future trends in Broadband Satellite communications: Information Centric Networks and Enabling Technologies
}

\author{
Tomaso De Cola ${ }^{1 *}$, Daniele Tarchi ${ }^{2}$, and Alessandro Vanelli-Coralli ${ }^{2}$ \\ ${ }^{1}$ Deutsches Zentrum fr Luft- und Raumfahrt (DLR), German Aerospace Center, Institute of Communications and \\ Navigation, Satellite Networks, Oberpfaffenhofen, 82234 Wessling (Germany) \\ 2 Department of Electrical, Electronic and Information Engineering, University of Bologna, Viale Risorgimento 2, \\ 40136 Bologna (Italy)
}

\begin{abstract}
SUMMARY
The ever-increasing demand for multimedia services from fixed and mobile users has motivated the scientific community, industries, and standardization fora to re-think the communication paradigm and the protocol architectures, resulting in an overall revolution of the Internet technologies. As satellite will play a pivotal role in this picture to exploit its broadcast and broadband capabilities, technical challenges come up in the integration of satellite and terrestrial networks. New paradigms based on the recent Future Internet studies findings have to be also applied to the SatCom domain, with consequent implications on the design of the physical layer, in order for the satellite capacity to accommodate all of the offered services. This paper explores the various challenges arising during this process and draws a new concept of broadband satellite systems, tailored to the needs of fixed and mobile users. Copyright (c) 2014 John Wiley \& Sons, Ltd.
\end{abstract}

Received ...

KEY WORDS: Future Internet, Information-Centric Networking, Satellite, Interference Management, Cognitive Radio

\section{INTRODUCTION}

The ever-increasing penetration of Internet in people everyday life has reshaped the classic concept of network architectures and motivated the design of new communication paradigms. In particular, it can be pointed out that the typical host-centric approach to design networks and services is going to be replaced by a content/information-centric design concept [1]. In more words, future Internet architectures are expected to be built on the basis of the services being transported and the specific requirements of quality of expectation and service demanded by the final users. In particular, the anytime-anywhere paradigm of nowadays data communication determined, on the one hand, a revolution of the applications' nature, which has become more pervasive, with the ultimate goal to let users be always online and causing a dramatic and unprecedented increase of the Internet volume of data, in the range of Zetabytes. This surge of data amount has pushed, on the other hand, the scientific community to re-engineer the current Internet in order to meet the needs of users and accommodate more efficiently the requested services.

Rethinking of network architectures resulted in the conception of the so-called Future Internet (FI) [2], which has been extensively investigated since early 2000's. In this framework, a large

*Correspondence to: Deutsches Zentrum fr Luft- und Raumfahrt (DLR), German Aerospace Center, Institute of Communications and Navigation, Satellite Networks, Oberpfaffenhofen, 82234 Wessling (Germany) 
number of project and research initiatives have been laid out, almost all yielding a number of competing networks architectures. Broadly speaking, two main, different, philosophies have been adopted to address this re-design task: evolutionary and revolutionary. The former aimed at provide new services and functionalities on top of the consolidated TCP/IP protocol suite and the latter at reinventing from scratch the protocol architecture. Independently of the specific line used in each architecture design, what is worth observing is the set of elements common to most of them: storage/caching, multicast, resilience, name-oriented data routing, and security.

The shift in the communication paradigm has not been limited only to network architectures' changes but also extended to the underlying technologies, because the advent of new services' typologies (e.g., cloud services, social networking, etc.) has required an enhancement of the transport capabilities at the physical layer. In more words, the increased capacity offered by wireless technology in local area networks along with the roll-out of $4 \mathrm{G}$ and the initial development of 5G [3] in cellular networks has broadened the range of services requested by end-users, especially for what concerns multimedia delivery. Further to this, mobility of users makes the access to content more widespread thanks to caching and relaying capabilities.

Along with the formidable technology advances experienced in the last years in the area of terrestrial communications, satellite is also expected to play a major role in delivering content because of the need to reach users without an efficient terrestrial infrastructure. Furthermore, the next frontiers of satellite communications is represented by the use of higher frequency bands in the feeder links, thus enabling a deeper penetration of services like High Definition Television (HDTV) and Ultra HDTV (UHDTV) [4], which request high-throughput links to meet real-time requirements. It is however immediate to see that terrestrial and satellite networks should not provide services to end users in parallel and in direct competition, but are expected to be bridged in order to increase the overall network transport capabilities and really implement the concept of anytimeanywhere Internet services, which sometimes is limited to some geographical areas because of cellular signal coverage and digital divide in general.

Integration of satellite and terrestrial networks obviously has diverse implications and introduces technical challenges from a networking and communication standpoint. As to the former, extension of information-centric networking (ICN) architectures [5] to satellite scenarios has to take into account the intrinsic capabilities of the technology (e.g., multicast and broadcast), but also the technical challenges in terms of link availability and propagation delays. Further to this, the integration demands specific coordination strategies between the various networked nodes involved in data delivery, so that recent advances in this areas have to be considered as as promising integration tool. To this aim a proper investigation of the mutual interference between different systems and communication links, and its management should be considered. Moreover, the coexistence of wireless and satellite links introduces technical issues related to the efficient use of spectrum, so that the advent of cognitive-radio based techniques seem particularly attractive to allow wireless and satellite networks to opportunistically exploit the available spectrum. Another enabling technology to be taken into account is instead represented by multi-antenna systems that allows to enhance the overall performance by introducing cooperative schemes among different nodes.

In response to the challenges presented above, this paper illustrates the main requirements that new Future Internet services are demanding, then showing the implications from a network architecture and communication capabilities perspective. In particular, the main contribution of the paper is to show how different elements of terrestrial and satellite networks can be bridged together to actually form the pillars of next-generation integrated networks. Particular attention will be actually devoted to the evolution of broadband satellite communication concepts, since adaption to terrestrial networks interfaces will be needed to achieve efficient system integration.

The remainder of this paper is structured as follows. Section II discusses Future Internet services with respect to the networking and communication requirements which have to be met in the network integration process. The implications from an information centric networking perspective are analyzed in Section III, whereas the communication counterpart is explored in Section IV. 
Finally, Section V draws the conclusion of the conducted study and indicates future lines of research for this framework.

\section{NEXT GENERATION SATCOM SCENARIOS AND REQUIREMENTS}

According to recent studies and industry forecast about satellite business [6], it is expected that the introduction of next-generation satellite networks will play a pivotal role in overall communications for the capability of complementing terrestrial networks and reaching users with broader coverage. Hence, the advantages provided by satellite communications won't be just an alternative to terrestrial communications where cable and fixed wireless infrastructures are not available but, more interestingly, will be the necessary glue between backbone and final users in order to provide new services according to an anytime-anywhere paradigm, thus, ultimately, achieving the goal of increasing the capacity of communication networks. To this regard, the features provided by nextgeneration communication networks will be supported by the broadcast/multicast capabilities of satellite networks on the one hand, and the widespread use of terrestrial technology to disseminate a huge amount of data. From this perspective, it is possible to identify a number of scenarios that see satellites as important element in the overall communication chain [7]:

1. IPTV services over heterogeneous broadcast networks. They consist of integrated satellite and terrestrial networks, where multihomed users' devices can receive data streaming from the available network interfaces, so to improve quality and interaction level.

2. Access to Internet services for institutional and professional applications from remote areas. It is about the governmental and business applications run from remote areas, e.g., oil platforms and isolated locations, where satellite technology is the only possible solution to access Internet and get connected with other sites.

3. Satellite broadband access for private applications from remote areas. It is the dual of the previous scenario, although the attention is here on the Internet access for private uses, implying less stringent measures from a security viewpoint.

4. Emergency and public safety communications. It concerns application scenarios where routine safety applications are enrolled or management of crisis situations is ongoing because of imminent or already occurred accidents (e.g., natural disasters or man-made events).

5. Heterogeneous mobile services. It is the case of the services requested by mobile users, possibly supported by both terrestrial and satellite connectivity.

6. $3 G / 4 G$ backhauling. It is about the integration of satellite with cellular infrastructures to help the penetration of native $3 \mathrm{G} / 4 \mathrm{G}$ services in areas where the deployment of terrestrial infrastructure is not feasible because of high costs or geomorphological limitations (e.g., mountains).

It is immediate to see that the aforementioned scenarios can be further merged according to the specific applications being considered and the use of heterogeneous technologies. In particular, throughout the rest of this paper we'll be mostly focused on the case of IPTV services (1), satellite broadcast for institutional purposes (2), and emergency communications (3), in order to better highlight the value of satellite in next-generation data dissemination networks for broadband applications. In particular, these three scenarios will be further elaborated within the next subsections in terms of capability requirements and in the next Section for what concerns the implications from an ICN architecture perspective.

Finally, a special note has to be reserved to those services which are not supposed to require broadband capabilities but are expected to grow in the near future. This is certainly the case of M2M applications [8], actually consisting in the distribution of large amount of data generated by actuators, collected by sensors and distributed to processing centers. In the light of their need of distributing a large amount of information to a high number of users, the use of multicast/broadcast communication paradigms offered by satellite communications is certainly a very attractive solution. Further to this, it must be observed that sensor applications, video-surveillance, and energy plant 


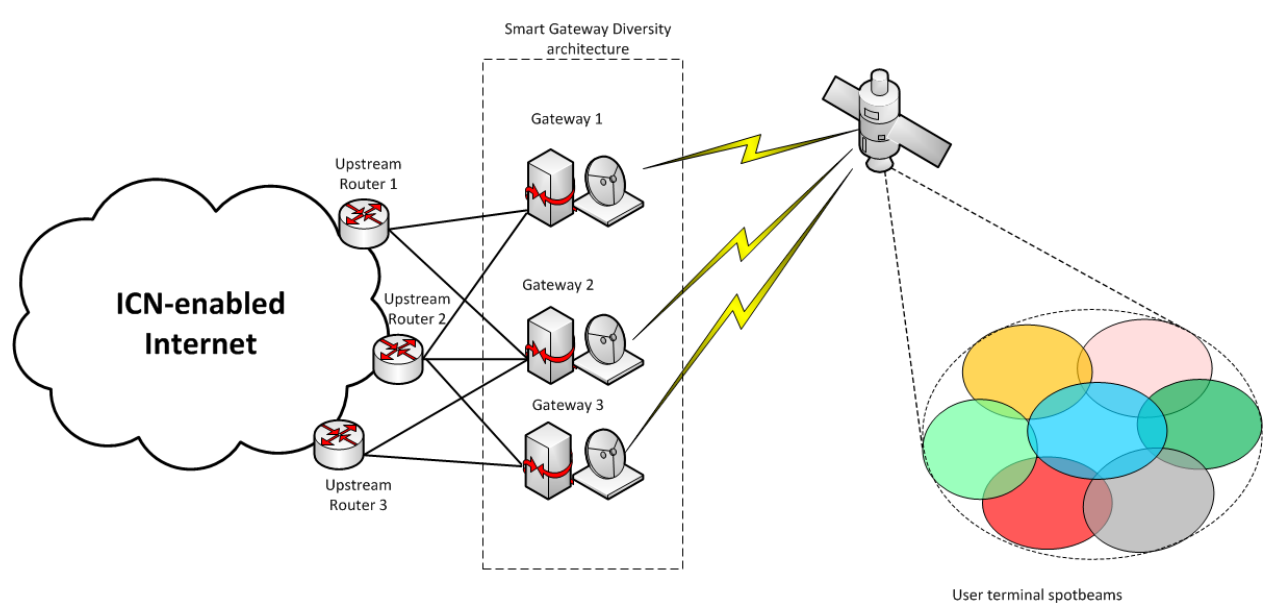

Figure 1. Smart-gateway diversity architecture with ICN support for IPTV services

control systems operates in areas where very often no terrestrial infrastructure is available, thus actually requiring satellite connectivity. Finally, if we consider the amount of data being transferred in these context and need for security and privacy solutions, the emergence of ICN architecture solutions will certainly have a primary role to make satellite communications more appealing also from content distribution perspective. Being not part of the focus of the present paper, the interested reader can refer to the relevant literature and in particular to $[9,10]$.

\subsection{IPTV services over heterogeneous networks}

Next-generation of satellite networks is expected to target Terabit/s capacity by employing higher frequencies $(\mathrm{Q} / \mathrm{V}$ bands) on the feeder link and building on smart-gateway diversity (SGD) architectures to cope with propagation impairments [11]. This network configuration is certainly the natural candidate for enabling high-quality video applications, such as HDTV, UHDTV and 3DTV, which require very high bit-rates and levels of Quality of Experience (QoE). On the other hand, it is worth noting that TV streaming is usually offered by terrestrial infrastructures by means of DVB-T/T2 technologies. The availability of multihomed devices building on the HbbTV standard can make better use of available network resources and also provide access to Internet services, thus making the user experience even more attractive from a market perspective. Example of a SGD architecture implementing ICN features is shown in Fig. 1.

Enabling such services over heterogeneous networks obviously introduces some requirements. As far as satellite is concerned, the use of spatial diversity techniques call for smart handover procedures to allow data re-routing from one gateway to another. Further to this, advanced interference mitigation techniques could be also beneficial to improve overall quality of service, which can be easily subject to degradation in UHDTV and 3DTV streaming. As far as the integration of terrestrial with satellite networks is involved, efficient paradigms to select the best route are necessary in order to optimally exploit the multihome capabilities of users' devices and to efficiently transport large amount of data. To this regard, the implementation of efficient content-aware routing strategies is of immediate need to achieve the QoS targets. Further to this, the broadcast/multicast capabilities intrinsic of satellite communications exploited together with the comcast functionalities offered by ICN architectures are key ingredient for a satisfactory fruition of IPTV services.

Hence, the resulting communication network, composed of integrated satellite and terrestrial links, has to meet specific requirements from both architecture capabilities and technologies features. 


\subsection{Satellite broadcast for professional and institutional services}

Providing connectivity between remote sites is a key-element in some governmental and professional applications, where various kinds of Internet services have to be provided in areas where no terrestrial infrastructures are available. This assumes some importance in the case of some company premises that need video/voice communication facilities as well as database distribution and synchronization functions thus requiring continuous and reliable connectivity with headquarters or main sites. This scenario is even more important in the case of institutional and military applications, where resilience of links against man-made disruptions has to be guaranteed and, further to this, effective security measures need also to be implemented. Further to this, distribution of data usually follows a multicast approach in order to make the same set of information available to different groups of users located in different premises, thus requiring an efficient distribution network.

To this regard, the availability of a satellite link is certainly a possible response to the aforementioned service requirements, provided that integration of ICN networks is also carried out in order to allow sites' connectivity and data distribution in a more efficient and secure way. In particular, issues related to security will assume a particular importance. On the one hand, enterprise-based solutions usually call for Virtual Private Network (VPN) oriented solutions so as to make secure the access and retrieval of data. On the other data, the sensitive data exchanged in governmental transactions or over military theaters require some more sophisticated security strategy. This issue becomes even more challenging if we take in consideration that nowadays solutions are usually built on key distribution centres, whose implementation and reachability can be not easy in these scenarios, where connectivity comes only by means of satellite. That's why moving to an ICN architecture paradigm is regarded as really helpful also to tackle these security issues.

\subsection{Satellite-based emergency communications}

Unavailability or partial disruption of terrestrial infrastructures is common in natural or man-made disasters, thus making the employment of satellite technology an absolute necessity. Further to this, communication and networking solutions able to exploit the limited connectivity in time and space are requested to let first responders and crisis management units to interact. From this perspective, design of such network has to be based on satellite technology, enriched of additional functionalities to meet the aforementioned needs. On the one hand, disruption tolerant networking (DTN) [12] architectures have to be taken as reference in order to allow exchange of sensitive data between the various players deployed on the field in spite of the lack of terrestrial infrastructure. To this regard, ad-hoc routing solutions and congestion control schemes aimed at optimally distribute the load over the available networks is of paramount importance. Further to this, it is immediate to see that deployment of traditional IP-based technologies based on end-to-end semantics can be successful in this scenario at the cost of some non-negligible workaround. By contrast, the use of ICN architectures implementing DTN concepts is certainly providing functionalities more in line with the peculiarities of the considered scenario, especially for what regards object naming and the related routing [13].

Requirements on the system design do not involve only architecture and networking aspects, but also entails some considerations at communication level. During the aftermath and later phases of a crisis situations, coexistence of satellite and ad-hoc wireless terrestrial infrastructure is taking place. As such, on the one hand opportunistic use of spectrum is certainly a key point to guarantee an effective exploitation of the available network resources. On the other hand, coordination between the different networked units (satellite terminals, base station, mobile devices) is recommended since centralized control of resources can be particularly challenging in these scenarios because of network fragmentation. 


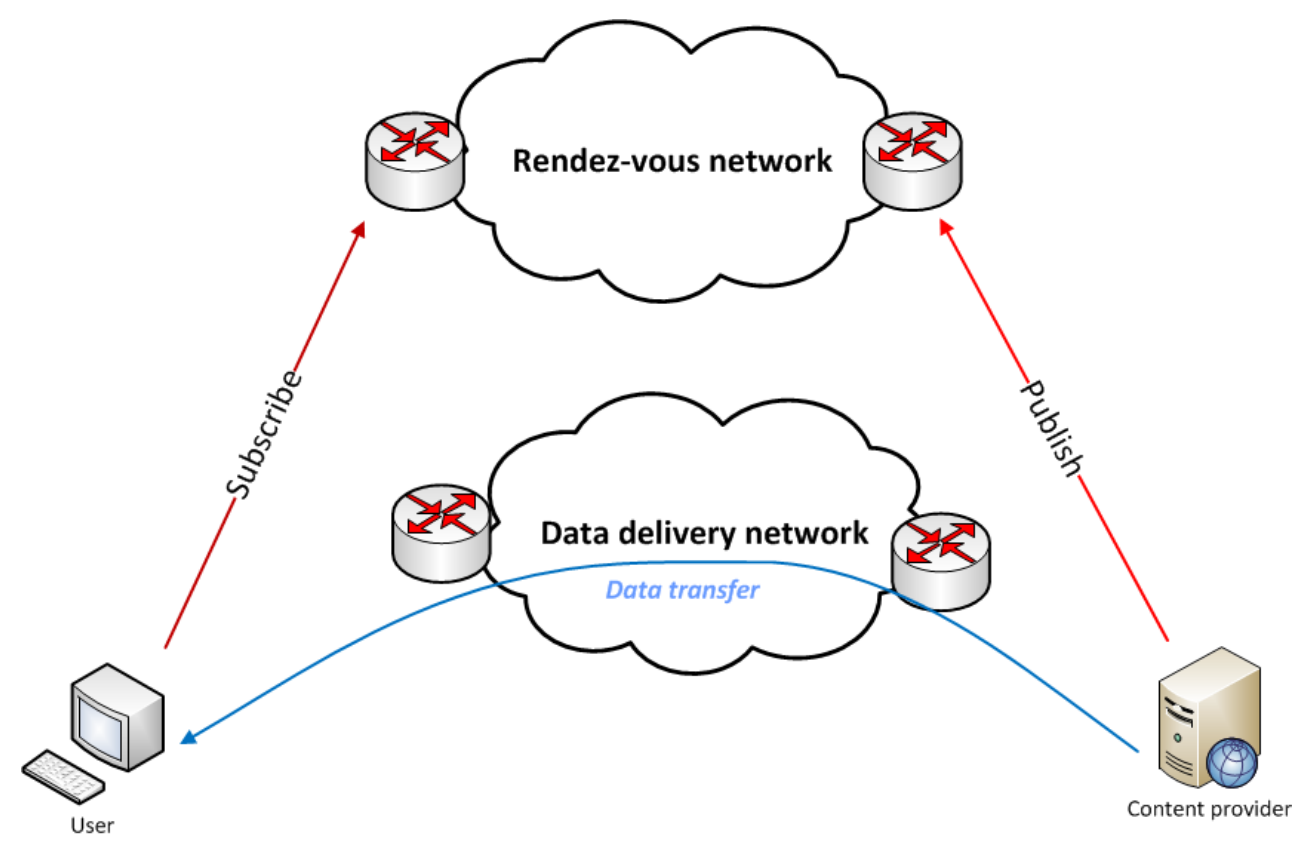

Figure 2. Example of pub-sub ICN architecture with rendez-vous points according to PURSUIT design

\section{ICN ARCHITECTURES}

Information-centric networking architectures represent a substantial shift from current Internet architectures in that they are host-centric whereas ICN aim at designing network around the content [14]. Being information the core of all network mechanisms, usual host addressing schemes (e.g., IP addresses) are replaced by object names, which in turn allow the implementation of contentaware routing schemes. Several exemplary architectures have been worked out over the last years, conceived to extend the concept of content delivery networks (CDN) and meet specific scenario requirements, such as the architectures developed in the PURSUIT [15], NDN [16], 4WARD [17], and SAIL [18] projects, just to cite a few.

Furthermore, the interaction between users and information also undergoes important changes. Users directly request the specific content they are interested into and made available from other users and content providers. As such, ICN architectures are built around publish-subscribe ( $p u b-s u b$ ) mechanisms, whereby signaling messages are exchanged beforehand to announce and then request specific content. Specific nodes, referred to as rendez-vous points in PURSUIT, are responsible for keeping status about the various requests and the locations where content is available, so that data transfer can be performed straightforwardly. The pub-sub philosophy is also powerful in enabling multicast communications, which usually require some workaround in native IP networks, which usually are not supporting multicast operations. According to the specific content requests performed by users, it is possible to aggregate requests and distribute contents to group of users. Obviously, the implementation of caches (i.e., storage units) on-path (i.e., available on the routing path) or off-path (i.e., available outside the routing path) further simplifies and accelerates data distribution without necessarily involving the content providers. The case of the PURSUIT architecture is sketched in Fig. 2, which exemplifies the pub-sub paradigm in general.

As outlined in the previous section, the reference scenarios introduce architecture and networking requirements that mainly concern security, broadcast/multicast capabilities, and efficient data distribution. As such, it has been observed that adoption of ICN architecture concepts already explored in past initiatives is a key point for the design of next-generation satellite networks. In particular the following elements, which are inherently supported in most ICN architectures, are expected to play a prominent role also in future satellite networks: 
- Name-based routing. Information centric network architecture makes a considerable shift from common host-centric architectures where addressing is performing through IP addresses. ICN, on the contrary, relies on object naming so that context/content awareness routing can be easily exploited.

- Distributed caching. Availability of on-path caching capabilities improves the accessibility to content and the retrieval, although the design has to take into account the characteristics of satellite networks, especially in terms of propagation delay that can affect the information refresh mechanisms.

- Disruption Tolerant Networking. Discontinuous connectivity in time causes networks to be fragmented, thus making the application of traditional IP-based routing schemes not profitable. DTN concepts, on the contrary, provide tools to compute the next-hop on hopby-hop basis without the need for establishing the entire path from sources to destinations.

- Hop-by-hop congestion control. Common to ICN and DTN architectures, the implementation of an overlay architecture running on top of technology-dependent layers allow to perform congestion and flow control on hop-by-hop basis, in order also to exploit caching capabilities implemented by the transport network.

Although the aforementioned concepts are quite general, their declination strictly depends on the specific scenario being considered, implying that different flavors of ICN architectures should be considered to meet the requirements of different scenarios. Hence, dedicated architecture design for the scenarios identified in the previous section is detailed in the following, where above ICN functionalities are considered separately along with other capabilities requested only by specific scenarios.

\subsection{IPTV services over heterogeneous networks}

As formerly introduced, distribution of IPTV services should be carried out by considering a smart gateway diversity architecture implementing ICN capabilities $[19,20]$ in order to better interwork with terrestrial networks and more efficiently distribute contents to users. Leveraging on the multihomed nature of end-users' devices, it is recommended that content can be disseminated over terrestrial and satellite links, whereas the signaling resulting from the pub-sub paradigm of ICN architectures should only transit over the terrestrial network in order to have a better interaction between users and providers for what concerns the requested services. For the sake of efficient delivery retrieval, on-path caching mechanisms should be also implemented throughout the entire network. A particular attention has to be dedicated to the satellite network in the light of the smart gateway diversity architecture. Caches should be implemented either on the satellite gateways or on the upstream routers, so that content can be retrieved directly from there also in case of gateway handover. If the former strategy is applied, it is possible to save some delay in signaling and content delivery, but at cost of increased storage units, which have to be implemented in all satellite network gateways. On the other hand, the implementation of caching capabilities certainly introduce some minor delay but is more efficient in terms of storage capacity throughout the entire network. It can be also observed that in some case upstream routers are actually gateways integrating the terrestrial and satellite network portion, so that caching capabilities on the last mile of the terrestrial infrastructure can be also collapsed in the upstream routers, thus making the overall caching architecture more efficient. To this regard, another important aspect relates on to how store content in the network. In order not to saturate the storage capacity, it is recommended to keep copy of the contents which have been required the most and to introduce an aging mechanism so that old content are finally removed from the cache. Further to this, the table of interest implemented in each ICN node should be updated also according to the propagation delay experienced by satellite network, which being much larger than that of terrestrial links can lead to unnecessary content update or premature removal from the caches.

Reliability can be ensured by means of network coding [21] implemented in the upstream routers and directly applied to the contents stored in the cache in order to save additional processing time due to the processing delay. Network coding would be particularly efficient here in order to 
cope with losses occurring as consequence of the gateway handover especially when the makebefore-break procedure is triggered too late and packets already in the transmission queue of the gateway will be lost. A further key element here is to apply DTN concepts in order to suspend and later resume the activity of a given gateway, by implementing strategies similar to custodial transfer specified in the Bundle Protocol [22]. Further to this, re-routing strategies implemented in preparation to handover events should be carried out so as to meet specific QoS requirements as already explored by the DTN scientific community, coming out with interesting results. Also linked to this point is the implementation of congestion/flow control mechanisms, which obviously follow a hop-to-hop strategy rather than common end-to-end principles as implemented in TCP/IP. This can help reduce the load on some satellite network gateway or even better distribute the overall load between satellite and terrestrial networks.

Finally, an important element that deserves a particular attention is the object naming strategy, which allows to implement content-based routing schemes. ICN scientific community has essentially worked out two possible main approaches towards object naming: flat and hierarchical names [5]. The former is string determined from hash computations, which can also embed a digital signature for the sake of content and source authentication. On the hand, the latter contains an indication about the hierarchy in an URI style, which can be exploited to link objects with specific repositories and caches, thus making content-based routing schemes more efficient. This approach, however, has some limitations because the large number of objects may cause high depths in the hierarchy, with object names becoming overly large for common caching systems. As such, an alternative approach would be to combine the flat and the hierarchical one, so as to take advantage of both, with small sacrifice in terms of storage capacity requirements.

\subsection{Satellite broadcast for professional and institutional services}

This scenario illustrates the cases where enterprise premises are located in remote areas and mutual interaction can be achieved only through satellite communications. Further to this, institutional services run over between these entities also require specific security measures, thus call for ICNbased solutions. As already described in the previous scenario, caching plays obviously a major role so as to avoid excessive data retrieval from source to destination. In this regard, another important requirement to be met is the multicast capability that be necessary in some applications aimed at delivering data only to some groups of users. To this end, the satellite technology certainly offers clears advantages but still ICN solutions are needed here to make the content distribution more efficient. In particular, the proper naming of objects can be particularly efficient to distribute data amongst the demanding users. Since data are exchanged mostly between remote entities, a hierarchical name approach seems more promising, as it can be also exploited to meet the multicast requirements. In particular, content destined to same group of users can be aggregated in a given level of the hierarchy, while the sublevels can point to the ICN nodes containing those data in the caches. In order to make the association between name and forwarding information basis (FIBs) more efficient, hierarchy should be defined according to Bloom filters [14], which have been explored by the recent ICN literature and recognized as particularly suited to these applications.

Another key element in these scenarios is the implementation of security measures. Usually institutional entities can exchange data over a VPN connection formerly established, in order to exchange credentials. This strategy, though quite effective and widespread, has the drawback in that it requires key distribution centres and quite some signaling traffic is exchanged between the involved parties. On the contrary, solutions proposed in the ICN context build on an information centric approach so that tunnels are actually not required and authentication can be already embedded in the object names or provided separately. In the latter case, particularly powerful is the application of identity based signatures (IBS) [19] applied together with encryption, so that source (identity) validation is performed first, and then decryption of the content is carried out according to specific signcryption algorithms [23]. In particular, the application of ElGamal or Schnorr's algorithms seems indeed suitable for these applications thanks to the limited size of the authenticated and encrypted messages and to the reduced processing time incurred in the validation process if compared to traditional IBS mechanisms. 


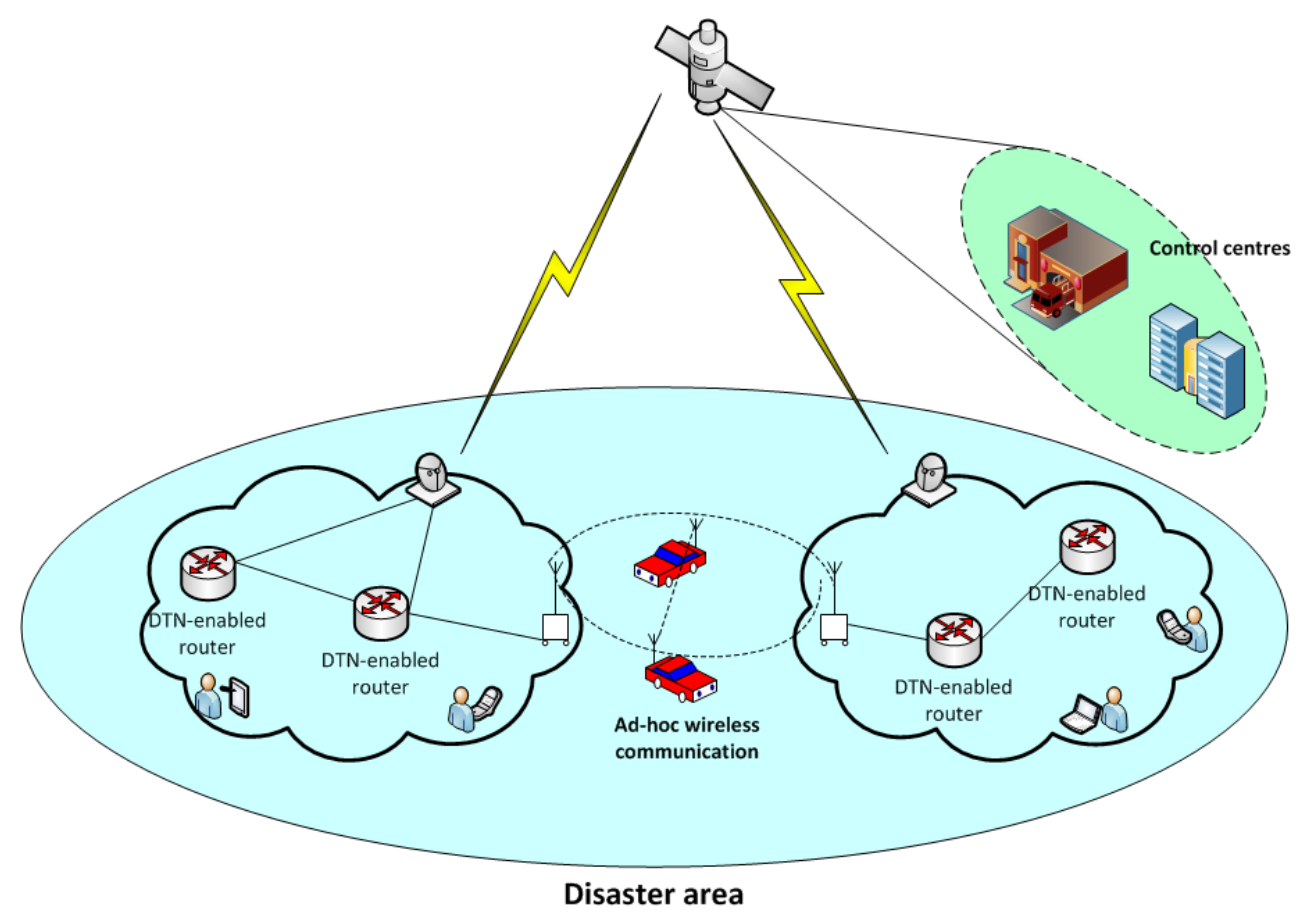

Figure 3. DTN-ICN-based architecture for emergency communications

\subsection{Satellite-based emergency communications}

Supporting emergency communication in disaster area is indeed a very complex task because terrestrial infrastructures are usually severely damaged or completely disrupted, whereby end-to-end path from source (e.g., injured people or first responders) and destination (e.g., public authorities, relief coordination centres) cannot be established. To overcome these service limitations, ad-hoc and temporary solutions are usually adopted. These solutions are actually working only under specific conditions (e.g., static network topology), so that minor fluctuations of the data traffic or sudden change of connectivity conditions as consequence of new link disruptions cannot be tolerated. These issues are mostly due to the fact that used networking paradigms still follow host-centric principles, whereas implementation of information-centric networking concept would help mitigates these problems.

First of all, application of DTN design concepts support data communication on hop-by-hop basis, thus overtaking the problem of temporary or sudden network disruptions. In this respect, suspending and resume of communication operations let information flow to be unaffected by connectivity unavailability and pushed data will be eventually available at destination as soon as a path between intermediate nodes and destinations can be established. In the light of the network instability, availability of caches is also of utmost importance, so that warning and alert messages, which are periodically re-disseminated, can reach the population at risk even in the case of no network path to control centres. The overall network architecture building on DTN and ICN concepts is shown in Fig. 3.

Secondly, as already emphasized in the other two reference scenarios, an important building block of ICN architecture [24] is to use object names rather than host addresses, thus allowing the implementation of content-based routing schemes. In general, since data stored in caches is indexed through Distributed Hash Tables (DHT), it is immediate to think of objects' names structured according to a hierarchical scheme, taking also into account that the number of networked nodes is limited and the frequency of alert message is somewhat low, whereby the size of FIBs is manageable.

Finally, a special note has to be dedicated to the role of available technologies. Satellite is certainly the most appropriate in these scenarios, as it is not subject to network disruption, can reach 
large areas and provides multicast/broadcast capabilities. Although satellite is certainly the only technology available during the first phases of the aftermath, it should be also noticed that temporary wireless terrestrial solutions are later deployed on the field to allow communications between first reponders and rescue teams, and eventually original terrestrial infrastructure is restored. From this picture it is therefore immediate to see that availability of multiple technologies should be exploited by multi-home communication paradigms, thus requiring the implementation of multi-path routing schemes which can be easily supported by ICN-based architectures. In more detail, routing is performed on hop-to-hop basis according to objects name, so that different next-hops can be selected for chunks composing the same object according to specific QoS metrics. For instance, being also congestion and flow control performed hop-by-hop, it is possible to select the next-hop according to the congestion/flow conditions experienced by the neighbour nodes.

\section{ENABLING TECHNOLOGIES}

In order to work in a proper way, ICN based SatCom solutions should implement advanced technologies at the lower layers. In the recent years, several lower layers technologies have been introduced aiming to improve system performance. One of the most challenging issues is the negative effect of the mutual interference that can be generated by communications belonging to the same system or communications belonging to different systems. The presence of interference reduces the overall capacity of the system limiting the potentialities of the ICN approach. Interference mitigation techniques should therefore be employed to reduce the mutual interference in communication systems [25].

In the following, the most promising interference reduction techniques are described focusing on their main characteristics and on the challenges we have to face when considering their implementation in SatCom scenarios.

Interference Management refers to strategies aimed at dealing with the ever-growing number of mobile devices to be served in next generation wireless systems, along with the parallel demand for increased capacity as a necessary condition to achieve the required transfer rates for the intended service (e.g., data transfer, voice-video, etc.). Solutions to such problem in terrestrial communications are declined into a plurality of techniques, which, according to scenarios peculiarities, are alternatively designed to avoid interference (Interference Avoidance - IA), to remove the existing interference (Interference Cancellation - IC), or to exploit the interference structure to increase the capacity (Multi-User Detection - MUD).

In the first category the concept of separating the interferers from the signal of interest, by properly designing the structure of the signals, is the key idea, which can then be accomplished in the spatial domain, i.e., with multi-antenna techniques, such as Dynamic Beamforming, in the sense of the Spatial Multiplexing of users according to the channel state through optimization algorithms, or in the signal domain, as in the case of Interference Alignment, in which all users coordinate their transmissions using a pre-coding scheme such that the overall interfering signal lies in a reduced subspace at each receiver: the aim is then to bring all interfering signals, at each intended receiver, onto a subspace with less dimensions (at least one less) than the transmitted signals space, since in such case decoding can be performed by considering the signal in the correct dimension of its relative subspace, which means ideally leaving out the interference.

The second category deals instead with the mitigation of the interference at the receiver side. Differently from the Interference Avoidance, in this case the interference is received at the terminal side and successively removed. This type of techniques includes both interference cancellation where the interferer signals are firstly detected and then eliminated for the signal of interest, or interference mitigation techniques employing the minimization of some metrics, e.g.,the squared errors, which include also blind techniques.

Finally, the third category, Multiuser Detection, addresses the exploitation of interference structure knowledge, i.e., interferers are treated as useful signals, to increase the overall capacity region. 
However, dealing directly with the other signal at the receiver or transmitter side is not the only method for decreasing its negative effect. One of the most interesting approaches raised in the last years is to consider a flexible spectrum usage that, exploiting the cognitive radio techniques based on the knowledge of the transmission of other systems, can define the optimal transmission parameters for reducing the mutual interference.

One of the most challenging trend in the next generation wireless system, is the coexistence of multiple systems within the same scenario. Thus, another interesting area of study is the design of smart solutions to cope with interference from heterogeneous wireless systems, which are highly relevant in todays very densely populated wireless environment. In this direction, more advanced strategies might be based on cognitive capabilities.

Most of the above mentioned techniques reside on the exploitation of advanced antenna solutions. In particular Multi User MIMO and Massive MIMO techniques seems to have the most favorable feedbacks from the research community as enablers for future generation services.

\subsection{Interference Avoidance}

The IA is a transmission strategy that drastically reduces the impact of interference. The basic concept behind it is to coordinate the transmission from multiple sources such that the mutual interferers can be aligned at the receivers, allowing easier interference cancellation. IA exploits the presence of multiple signalling dimensions in terms of time slots, frequency blocks and antennas. The transmitters jointly designs their transmitted signals over all dimensions in such a way that at each receiver the interference occupies only a portion of the overall signal space. This has been proven to yield the very interesting result that the sum rate of a network might grow linearly and unbounded with the size of the network itself, in clear contrast with orthogonal strategies such as FDMA or TDMA, where the sum rate is basically constant with respect to the network size since only two users can communicate in each resource block.

In its most straightforward version, IA is a sort of precoding scheme applied to the interference channel, by which signals are linearly encoded over time, frequency and possibly space (multiantenna case). In this way the interfering contributions aggregated at each receiver are observed into a low dimensional subspace of the overall signals space, and the number of non-interfering symbols that can be transmitted over the interference channel is maximized.

Open challenges remain for IA, which, for instance, relies on strong assumptions which must be formulated in more relaxed versions in order for this technique to be implemented in practical system such as the dimensionality of alignment, which has been shown to grow faster than exponentially in some cases with the number of users, and this is only relatively mitigated in the case of MIMO IA.

IA relies strongly on Channel State Information (CSI) and synchronization in order to calculate the proper precoders values. In this sense, research efforts need to be focused also on low overhead signaling or feedback strategies for CSI and synchronization strategies, even leveraging on GPS satellite signals.

IA techniques could be a viable option in SatCom, especially in multibeam communication systems, where they have been proposed for reducing the interference at the satellite receiver in the reverse link [26]. By using IA techniques it would be possible to have a higher number of ground terminals communicating with multibeam satellite stations. IA has been also proposed for reducing the interference generated in a cognitive satellite scenario where a multibeam secondary satellite system employing IA techniques can reduce the interference toward another satellite system operating on the same frequency bands [27].

\subsection{Interference Cancellation}

Differently from IA, the interference cancellation is based on a postprocessing approach aiming to eliminate or decreasing the detrimental effect of the interference caused by other sources and/or users. 
To this aim, in the literature, the IC issues can be approached by considering that the interference may be caused by other users/signals belonging to the same system, leading to an intra-system interference, or users/signals belonging to other systems, leading to inter-system interference.

The main difference between the two cases, is that in intra-system interference it is possible to know some parameters of the interfering source and exploit them for enhancing the IC performance. Indeed, the IC techniques are also often referred as Multi-User Detection (MUD) technique for their ability in discerning and eliminating the mutual interference of different users.

Even if optimal IC techniques have been proposed, depending on the multiple access scheme used in the specific system, its cost is prohibitive especially when the users/signals increase in numbers. Hence suboptimal approaches have been proposed aiming to reduce the mutual interference of the users. The suboptimal IC receivers can be divided into two main categories, linear and non-linear approaches [28].

The linear approaches (e.g., the Minimum Mean Squared Error - MMSE - approach) aim at reducing the mutual interference subject by each user by minimizing the interference signal effects; these techniques are mainly implemented by using iterative approaches.

Differently non-linear approaches aim at reducing the mutual interferences by detecting and deleting the signal from the others. Within this context the algorithms can be roughly divided into two main categories, Parallel and Successive Interference Cancellation. If Parallel Interference Cancellation aims at detecting all the signals together and then deleting them from the others, in order to implement a following detecting stage, in the Successive Interference Cancellation, the idea is to detect the more reliable signal and then delete it from the overall signal, and so on until the weakest signal.

More efficient schemes involving a joint Successive and Parallel Interference Cancellation have been also proposed aiming at detecting a set of more reliable signals in parallel and then applying the successive interference cancellation to the reminder.

The aforementioned IC techniques can be applied to different systems, depending on the selected access scheme. As a reference, IC can be applied to CDMA, OFDMA and TDMA systems, all characterized by the presence of multiple users/signals interfering among them.

In case of satellite communications, the IC techniques are also promising allowing to detect in a more reliable way the signals from both ground and satellite stations. In particular, for the SatCom, the IC techniques become more challenging due to the presence of multibeam scenarios, characterized by the the presence of multiple beams covering a certain area. In this case the mutual interference, also called co-channel interference, could occurs [29]. Due to the system nonlinearities also adjacent channel interference between near beams operating at adjacent frequency bands could occurs. Also in this case the use of IC techniques allows to increase the system performance.

\subsection{Multiuser Detection}

MUD techniques have been successfully addressed in terrestrial systems for many years, and already studied for Satellite systems at the gateway side, i.e., on the return link [30]. Recently, several studies have been initiated to assess the applicability of MUD techniques at the user receiver side [31] in order to increase the forward link capacity, that, in several applications represents the bottleneck of the system. Contrary to terrestrial systems, MUD implementation in satellite systems is not straightforward and introduces significant challenges both from the communication theory and hardware complexity point of view. In particular, a key challenges that is still to be fully addressed for MUD at the user receiver, is channel synchronization and estimation operations that have to be performed at extremely low SNRs. To this aim, frame structures considering the specificity of the satellite channel have to be devised to provide the hooks for synchronization and estimation algorithms in interference limited asynchronous scenarios.

\subsection{Flexible Spectrum Usage}

One of the key element for future communication systems is the opportunistic spectrum access, characterized by the presence of secondary users that after identifying unused spectrum bands, at 
a given time and place, are able to use them while not generating harmful interference to primary license holders. The flexible spectrum usage is at the base of the mitigation of mutual interference among different communication systems, by employing the so called cognitive radio approach.

The usage of radio spectrum resources and regulation of radio emissions are coordinated by national regulatory bodies such as the Federal Communications Commission (FCC), that assigns spectrum to licensed holders, also known as primary users. A large portion of assigned spectrum is used sporadically; the spectrum usage is concentrated on certain portions of the spectrum while a significant amount of the spectrum remains under-used or unused.

This principle, exploiting the temporary unused frequencies, has been firstly introduced by Joseph Mitola in 1999 [32] and is called cognitive radio (CR). The definition of the cognitive radio adopted by FCC is: Cognitive radio: A radio or system that senses its operational electromagnetic environment and can dynamically and autonomously adjust its radio operating parameters to modify system operation, such as maximize throughput, mitigate interference, facilitate interoperability, access secondary markets.

As the primary users have priority in using the spectrum, an important functionality that a cognitive radio device has to implement is the capability to work without interfering with the existing users. The main features of a cognitive radio are cognitive capability and reconfigurability. Cognitive capability refers to the ability to sense and gather information from the surrounding environment, so that the portions of the spectrum that are unused at a specific time and location can be identified.

Reconfigurability refers to the ability of rapidly adapting the operational parameters according to the sensed information in order to achieve the optimal performance. New emerging cognitive radio paradigm, hence, allow radios to opportunistically transmit in the vacant portions of the spectrum, also known white spaces, already assigned to licensed users, without interfering with them.

$\mathrm{CR}$ can facilitate interoperability between various communication systems. Through adapting to the requirements and conditions of another network, the CR devices can support multiple service types, such as voice, data, and video.

An important requirement of the cognitive radio network is the ability to sense the white spaces. Spectrum sensing is the task of obtaining awareness about the spectrum usage and existence of primary users in a geographical area. A relevant issue in spectrum sensing is the sensing periodicity: while utilizing a white space, the secondary system should continue to periodically sense the band in case primary user starts to transmit. The sensing period determines the maximum time during which the secondary user will be unaware of a reappearing primary user and hence may harmfully interference with it. In general the sensing period depends on the type of the primary service. For instance, one expects the sensing period to be very small for public safety spectrum, while less frequent sensing may be allowed for TV spectrum where the spectrum usage varies over a much large timescale.

After detecting the characteristics of the environment, a secondary user is allowed to transmit data simultaneously with a primary user or alone when the primary users are not using the spectrum. In the first case, the interference temperature limit should not be violated, while in the second one, the secondary user can transmit until the primary user needs the frequency. The CR has to be aware of primary users reappearance through various detection techniques, such as energy detection, feature detection, matched filtering and coherent detection. Detection performance of single user sensing, while scanning for primary users transmissions, can be pretty limited by many factors like noise uncertainty, shadowing and multipath effect. To overcame to this problem, a cooperative sensing can be used for improving detection accuracy by taking advantage of spatial and multi-user diversity.

4.4.1. Heterogeneous Networks Spectrum Sharing A challenging topic with an increasing interest is the possibility to deploy a HetNet scenario taking into account cognitive radio technologies. By focusing on a terrestrial wireless scenario, femtocells offer a promising solution for indoor communications. Femtocells are low-power, low-cost, short-range, plug-and-play cellular BS deployed in a residential area or small office. Femtocells enhance system capacity and coverage via improving link quality and enabling spectral reuse. In femtocells, higher data rates and better 
coverage are achieved by shorter communication distance and less penetration loss. Furthermore, high cellular capacity is reached by efficient spatial spectrum reuse in a smaller cell size. Femtocells not only provide better quality of service $(\mathrm{QoS})$ to indoor users, but also diminish site expenditure, maintenance cost, and power consumption of base stations.

Nowadays femtocells have attracted a lot of attention from both academia and industry. One of the most challenging issues in the femtocells deployment is the channel assignment: actually there are three options: dedicated-channel deployment, partial channel-sharing deployment, and co-channel deployment. Co-channel deployment is more attractive to operators due to low cost and backward compatibility. However, co-channel deployments of closed subscriber group (CSG) femtocells create coverage holes in macrocells. In order to solve this problem, cognitive femtocells are developed to sense their surroundings and flexibly adapt their operations to minimize interference. In the cognitive paradigm, interference mitigation approaches can be classified as opportunistic interference avoidance, interference cancellation, and interference alignment. Femtocells are overlaid within macrocells in a two-tier heterogeneous network. Allocating spectrum resources between femtocells and macrocells is a very important issue.

The increasing interest towards femtocells deployment has carried also their introduction in the SatCom world, where terrestrial and satellite links can be considered as part of the macro and femtocells channel deployment problem. To this aim the channel deployment techniques above described are under consideration for their extension toward a hybrid satellite-terrestrial scenario.

4.4.2. SatCom Cognitive Radio There is a long-standing interest in making radio systems more cognitive. Aiming to achieve a higher level of flexibility adapting themselves to changes in the environment and finding out how could exploit radio resources in a more efficient way, Cognitive Radios frequently claim to be the solution to the spectrum scarcity problem. Although large studies and research to apply these concepts and techniques in terrestrial systems during the past years, the interest in providing a more reliable cognition level also for satellite systems is growing up [33].

Since the first appearance of the term cognitive associated with a radio system in [32], only in the last few years due to the need to better resources exploitation for broadband services and thanks to developments in the digital communication field, it seems affordable talking about cognitive radio and satellite communications. However, satellite communications represent a scenario with specific requirements that need to be taken into account; the development of such techniques in a satellite environment need an in-depth evaluation or a description from a different point of view.

The state of the art about the applicability of cognitive radio techniques in satellite environments is presented in [34], whereas in [35] and [36] the focus is on challenges, scenarios, and techniques. Most of the works consider the problem of defining general scenarios where it is possible to implement cognitive techniques while only few deal with specific solutions or assessments of their applicability.

One of the main challenges to be faced considering the application of cognitive techniques in satellite environments is due to the inherit nature of satellite communications. SatComs are essentially characterized by the presence of a full area coverage with very low power at the earth ground, mainly due to the very high distance of the satellite transmitter. On the other hand spectrum is generally shared with other independent terrestrial links. In particular, by focusing on microwave frequency bands, the coexistence should be between satellite and terrestrial microwave links, characterized by point-to-point communications and very directive antennas.

A particular attention has been put recently in the exploitation of $\mathrm{Ku}$ and $\mathrm{Ka}$ bands arising from the demand for deployment of new services in these bands, CEPT has established through Decisions $\mathrm{ERC} / \mathrm{DEC} /(00) 07$ and ECC/DEC/(13)01 that coexistence between Fixed Satellite Services (FSS) and Fixed Services (FS) could be possible.

In these bands, according to the Radio Regulation provisions, FSs are usually allocated as primary users [37, 38]. In most cases Point-to-Point microwave links are established and hence in our scenario working in the band between 17.7 and $19.7 \mathrm{GHz}$ we shall consider such types of services as primary users. 
Among others the CoRaSat project [33] is working toward the definition of suitable solutions for allowing the spectrum sharing among different satellite services and among satellite and terrestrial services with a special attention toward the $\mathrm{Ku}$ and $\mathrm{Ka}$ bands due to their popularity for both operators and users in bringing new services.

\subsection{Multiple Antennas and Massive MIMO}

Multi-antenna techniques started being studied two decades ago, with the landmark papers on MIMO capacity and on layered space-time transmission. Then the studies evolved from the analytic characterization of the achievable rates to actual implementation schemes. Indeed, terrestrial communication standards, both broadcast and point-to-point, are making use of multi-antenna techniques to enhance the throughput or the robustness of the system. In the past few years, new techniques have been proposed, mainly based on multi-user transmission and cooperation. Such techniques allow the creation of a virtual MIMO (if spatially separated single-antenna devices are allowed to cooperate) or the reduction of the negative effects of the multi-antenna environment (in case of precoding).

Since several years the applicability of MIMO techniques in Satellite Communication has an increasing interests; however, some challenges still remain [39]. Moreover, due to the specific characteristics of the satellite communications some MIMO techniques have been specifically developed. Among others we can recall those exploiting the presence of dual polarized communications [40], typical of SatCom, and those involving multiple satellites [41].

A particular interest is towards those multi-antenna techniques that foresee to exploit multiple nodes both in transmission and reception. In that sense MultiUser MIMO (MU-MIMO) [42] and Coordinated Multipoint (CoMP) [43] represent two outstanding schemes to be considered.

MU-MIMO is widely considered a key technology for system capacity improvement in modern wireless networks. In contrast to Single User MIMO, where the spatial multiplexing gain is confined to a single user, MU-MIMO allows multiple users to be co-scheduled on the same time-frequency resources to exploit this gain among two or more terminals. This is particularly beneficial as high-rank SU-MIMO transmission is often limited by the number of antennas and antenna design constraints at the user end, whereas high-rank transmission using MU-MIMO is more feasible due to the scattered user distribution.

On the other hand, Coordinated multipoint (CoMP) transmission and reception techniques utilize multiple transmit and receive antennas from multiple antenna site locations, which may or may not belong to the same physical cell, to enhance the received signal quality as well as decrease the received spatial interference.

SU-MIMO transmissions occur in time-frequency resources dedicated to a single-terminal user equipment, and allow achieving the peak user spectral efficiency. They encompass techniques ranging from transmit diversity to spatial multiplexing and beamforming. On the other hand MUMIMO allocates multiple users in one time-frequency resource to exploit multi-user diversity in the spatial domain, which results in significant gains over SU-MIMO, especially in spatially correlated channels.

Hence, MU-MIMO, together with architectures, opens up novel possibilities in better sharing of resources among users. Efficient use of MU-MIMO, however, presents some nontrivial challenges: MU-MIMO has an inherit cost at physical layer depending on the type and degree of cooperation. Specifically, for many MU-MIMO techniques CSI must be known at the transmitter with sufficient accuracy in order to enable efficient cooperative transmission.

Since MU-MIMO primarily relies on spatial domain separation for user multiplexing, the environments with sufficient user separation are most suitable.

CoMP has been instead introduced for improving the performance on the LTE roadmap beyond Release 9. In LTE Release 11, some simpler CoMP concepts may appear, but it is generally expected that advanced CoMP concepts will take longer to be mature enough for commercial use [43].

One of the key elements of mobile radio networks is the spatial reuse (i.e., the reuse of resource elements such as timeslots or frequency bands) in a geographical distance, where the signal strength is reduced due to path loss, shadowing, and so on. 
Historically, this was achieved using network planning with certain frequency reuse patterns, which have, however, the drawback of poor resource utilization. $3 \mathrm{G}$ and $4 \mathrm{G}$ technologies are using full frequency reuse, which in turn leads to interference between the cells.

Network coordination has been presented as an approach to mitigate intercell interference and hence improve spectral efficiency. The same spectrum resources are used in all sectors, leading to interference for terminals at the edge between the cells, where signals from multiple base stations are received with similar signal power in the downlink. Multiple sectors of one base station can cooperate in intrasite CoMP, whereas intersite CoMP involves multiple Base Stations.

The sectors at one site can be different self-sustained units, or different remote radio heads linked via fiber to a central baseband unit. The Base Stations may be interconnected by the logical interface. The cooperation techniques aim to avoid or exploit interference in order to improve the cell edge and average data rates. CoMP can be applied both in the uplink and downlink. All schemes come with the cost of increased demand on backhaul (high capacity and low latency), higher complexity, increased synchronization requirements, more channel estimation effort, more overhead, and so on.

The use of CoMP in SatCom is still in an infancy state [44], mainly because CoMP needs of a strict coordination among different sites. Moreover a big amount of traffic should be exchanged among the different sites for coordinating them and/or joint process the user traffic.

Another interesting trend is represented by the Massive MIMO [45]. Massive MIMO is a particular type of Multiuser MIMO characterized by a very large number of antennas at the BS side, much larger with respect to the number of users. Thanks to this huge number of antennas, it is possible to achieve a very high directivity giving rise to high multiplexing gains. Under the hypothesis of uncorrelation among the different antennas, it is possible to demonstrate that the communication channels are orthogonal among them, leading to very simple linear processing.

The use of Massive MIMO in satellite communications is still in an unexplored while challenging area, due to the advantages it can bring. However, it is limited by the difficulties in installing a huge number of antennas at the satellite side [46].

\section{CONCLUSIONS}

This paper surveyed the potentials of ICN architectures for next-generation satellite networks in a number of relevant scenarios. The main features of information-centric approaches help more easily integrate terrestrial and satellite networks, thus also offering better quality of experience perceived by users. In particular, the different scenarios considered in the paper showed as use of DTN-based concepts along with object naming and identity security tools can definitely improve services data distribution even in the case of network disruption.

The paper also showed that advantages provided by ICN architectures have to be in any case supported by the underlying technology in order to meet the service and network requirements introduced in the reference scenarios. As such, the opportunistic access to spectrum performed by means of cognitive radio-based strategies emerged as a key point to ease the integration between satellite and terrestrial networks as well as interference management, deemed necessary to allow coexistence between different technologies. Finally, the need for non-centralised access to channel resources has showed the benefits possibly coming from CoMP techniques, which are regarded as particularly promising in scenarios where decentralised control of resources is the only feasible design solution (e.g., emergency communications).

\section{REFERENCES}

1. Mathieu B, Truong P, You W, Peltier J. Information-centric networking: a natural design for social network applications. Communications Magazine, IEEE July 2012; 50(7):44-51, doi:10.1109/MCOM.2012.6231278.

2. Stuckmann P, Zimmermann R. European research on future internet design. Wireless Communications, IEEE October 2009; 16(5):14-22, doi:10.1109/MWC.2009.5300298.

3. Chen S, Zhao J. The requirements, challenges, and technologies for $5 \mathrm{~g}$ of terrestrial mobile telecommunication. Communications Magazine, IEEE May 2014; 52(5):36-43, doi:10.1109/MCOM.2014.6815891. 
4. Sugawara M, Masaoka K. Uhdtv image format for better visual experience. Proceedings of the IEEE Jan 2013; 101(1):8-17, doi:10.1109/JPROC.2012.2194949.

5. Ahlgren B, Dannewitz C, Imbrenda C, Kutscher D, Ohlman B. A survey of information-centric networking. IEEE Communications Magazine July 2012; 50(7):26-36, doi:10.1109/MCOM.2012.6231276.

6. Agyapong P, Sirbu M. Economic incentives in information- centric networking: implications for protocol design and public policy. Communications Magazine, IEEE December 2012; 50(12):18-26, doi:10.1109/MCOM.2012. 6384447.

7. Siris V, Ververidis C, Polyzos G. Techno-economic gains analysis of services over an information-centric integrated satellite-terrestrial network. Future Network and Mobile Summit (FutureNetworkSummit), 2013, 2013; 1-10.

8. Kim J, Lee J, Kim J, Yun J. M2m service platforms: Survey, issues, and enabling technologies. Communications Surveys Tutorials, IEEE First 2014; 16(1):61-76, doi:10.1109/SURV.2013.100713.00203.

9. Duke-Woolley R. Findings from esa samos project - satellite $\mathrm{m} 2 \mathrm{~m}$ observatory study. Advanced Satellite Multimedia Systems Conference (ASMS) and 12th Signal Processing for Space Communications Workshop (SPSC), 2012 6th, 2012; 13-20, doi:10.1109/ASMS-SPSC.2012.6333066.

10. K Liolis GCP V A Siris. On satellite-assisted publish-subscribe future network architectures for smart $\mathrm{m} 2 \mathrm{~m}$ applications. 6th Future Internet Cluster Workshop, Network Architecture for a Smarter Environment, 2011.

11. Jeannin N, Castanet L, Radzik J, Bousquet M, Evans B, Thompson P. Smart gateways for terabit/s satellite. International Journal of Satellite Communications and Networking 2014; 32(2):93-106, doi:10.1002/sat.1065. URL http://dx.doi.org/10.1002/sat.1065.

12. Fall K, Farrell S. Dtn: an architectural retrospective. Selected Areas in Communications, IEEE Journal on June 2008; 26(5):828-836, doi:10.1109/JSAC.2008.080609.

13. Pentikousis K, Ohlman B, Corujo D, Boggia G, Tyson G, Davies E, Molinaro A, Eum S. Information-centric Networking: Baseline Scenarios. draft-irtf-icnrg-scenarios-03. URL https://datatracker.ietf.org/ doc/draft-irtf-icnrg-scenarios/.

14. Xylomenos G, Ververidis C, Siris V, Fotiou N, Tsilopoulos C, Vasilakos X, Katsaros K, Polyzos G. A survey of information-centric networking research. Communications Surveys Tutorials, IEEE Second 2014; 16(2):10241049, doi:10.1109/SURV.2013.070813.00063.

15. Giannaki V, Vasilakos X, Stais C, Polyzos G, Xylomenos G. Supporting mobility in a publish subscribe internetwork architecture. Computers and Communications (ISCC), 2011 IEEE Symposium on, 2011; 1030-1032, doi:10.1109/ISCC.2011.5983977.

16. Corujo D, Aguiar RL, Vidal I, Garcia-Reinoso J. A named data networking flexible framework for management communications. Communications Magazine, IEEE December 2012; 50(12):36-43, doi:10.1109/MCOM.2012. 6384449.

17. Hasan M, Amarasinghe H, Karmouch A. Network virtualization: Dealing with multiple infrastructure providers. Communications (ICC), 2012 IEEE International Conference on, 2012; 5890-5895, doi:10.1109/ICC.2012. 6364756.

18. Zhao L, Zaki Y, Udugama A, Toseef U, Gorg C, Timm-Giel A. Open connectivity services for future networks. Emerging Technologies for a Smarter World (CEWIT), 2011 8th International Conference Expo on, 2011; 1-4, doi:10.1109/CEWIT.2011.6135865.

19. Detti A, Caponi A, Blefari-Melazzi N. Exploitation of information centric networking principles in satellite networks. Satellite Telecommunications (ESTEL), 2012 IEEE First AESS European Conference on, 2012; 1-6, doi:10.1109/ESTEL.2012.6400110.

20. Galluccio L, Morabito G, Palazzo S. Caching in information-centric satellite networks. Communications (ICC), 2012 IEEE International Conference on, 2012; 3306-3310, doi:10.1109/ICC.2012.6363993.

21. Muhammad M, Giambene G, de Cola T, Berioli M, Alagha N. Network-Coding-Based Gateway Handover Scheme for Terabit Satellite Networks. 31st AIAA International Communications Satellite Systems Conference (ICSSC), Florence, Italy, 2013.

22. Scott K, Burleigh S. Bundle Protocol Specification. RFC 5050 (Experimental) Nov 2007. URL http://www . ietf.org/rfc/rfc5050.txt.

23. Galindo D, Garcia FD. A schnorr-like lightweight identity-based signature scheme. AFRICACRYPT, 2009; 135148.

24. Tyson G, Bodanese E, Bigham J, Mauthe A. Beyond content delivery: can icns help emergency scenarios? Network, IEEE May 2014; 28(3):44-49, doi:10.1109/MNET.2014.6843231.

25. Jiang L, Vázquez-Castro MA. Interference management versus interference cancellation: SATCOM case. Personal Satellite Services, Lecture Notes of the Institute for Computer Sciences, Social Informatics and Telecommunications Engineering, vol. 71, Giambene G, Sacchi C (eds.). Springer Berlin Heidelberg, 2011; 260-273, doi:10.1007/ 978-3-642-23825-3_24. URL http://dx. doi.org/10.1007/978-3-642-23825-324.

26. Jain P, Vazquez-Castro M. Subspace interference alignment for multibeam satellite communications systems. Proc. of 5th ASMS and 11th SPSC, 2010; 234-239, doi:10.1109/ASMS-SPSC.2010.5586887.

27. Sharma SK, Chatzinotas S, Ottersten B. Interference alignment for spectral coexistence of heterogeneous networks. EURASIP Journal on Wireless Communications and Networking 2013; 2013(46), doi:10.1186/ 1687-1499-2013-46.

28. Miridakis N, Vergados D. A survey on the successive interference cancellation performance for single-antenna and multiple-antenna OFDM systems. IEEE Communications Surveys Tutorials First 2013; 15(1):312-335, doi: 10.1109/SURV.2012.030512.00103.

29. Millerioux JP, Boucheret ML, Bazile C, Ducasse A. Iterative interference cancellation and channel estimation in multibeam satellite systems. International Journal of Satellite Communications and Networking 2007; 25(3):263283, doi:10.1002/sat.875. URL http://dx.doi.org/10.1002/sat. 875.

30. Lombardo F, Vanelli-Coralli A, Candreva EA, Corazza GE. Throughput distribution analysis of return link multigateway interference cancellation strategies for multi-beam broadband satellite systems. Proc. of 2012 IEEE Globecom Workshops (GC Wkshps), 2012; 95-99. 
31. Andrenacci S, Angelone M, Candreva EA, Colavolpe G, Ginesi A, Lombardo F, Modenini A, Morel C, Piemontese A, Vanelli-Coralli A. Physical layer performance of multi-user detection in broadband multi-beam systems based on DVB-S2. Proceedings of 20th European Wireless Conference, Barcelona, Spain, 2014.

32. Mitola J, Maguire J G Q. Cognitive radio: making software radios more personal. IEEE Personal Communications Aug 1999; 6(4):13-18.

33. Liolis K, Schlueter G, Krause J, Zimmer F, Combelles L, Grotz J, Chatzinotas S, Evans B, Guidotti A, Tarchi D, et al.. Cognitive radio scenarios for satellite communications: The CoRaSat approach. Proc. of FuNeMS 2013, Lisboa, Portugal, 2013.

34. Sharma SK, Chatzinotas S, Ottersten B. Satellite cognitive communications: Interference modeling and techniques selection. Proc. of 6th ASMS and 12th SPSC, Baiona, Spain, 2012.

35. Tarchi D, Guidotti A, Icolari V, Vanelli-Coralli A, Sharma SK, Chatzinotas S, Malekil S, Evans B, Thompson P, Tang W, et al.. Technical challenges for cognitive radio application in satellite communications. Proc. of 20149 th International Conference on Cognitive Radio Oriented Wireless Networks and Communications (CROWNCOM), Oulu, Finland, 2014; 136-142.

36. Hoyhtya M, Kyrolainen J, Hulkkonen A, Ylitalo J, Roivainen A. Application of cognitive radio techniques to satellite communication. Proc. of IEEE DYSPAN 2012, 2012; 540-551.

37. The European table of frequency allocations and applications in the frequency range $8.3 \mathrm{kHz}$ to $3000 \mathrm{GHz}$ (ECA table). ERC Report 25, ECC/CEPT Oct 2013. URL http://www.erodocdb.dk/Docs/doc98/ official/pdf/ercrep025.pdf.

38. ITU Radio Regulations 2012.

39. Arapoglou P, Liolis K, Bertinelli M, Panagopoulos A, Cottis P, De Gaudenzi R. MIMO over satellite: A review. IEEE Communications Surveys Tutorials First Quarter 2011; 13(1):27-51, doi:10.1109/SURV.2011.033110.00072.

40. Liolis K, Gomez-Vilardebo J, Casini E, Perez-Neira A. Statistical modeling of dual-polarized MIMO land mobile satellite channels. IEEE Transactions on Communications Nov 2010; 58(11):3077-3083, doi:10.1109/TCOMM. 2010.091710.090507.

41. Liolis KP, Panagopoulos AD, Cottis PG. Multi-satellite MIMO communications at Ku-band and above: Investigations on spatial multiplexing for capacity improvement and selection diversity for interference mitigation. EURASIP Journal on Wireless Communications and Networking 2007; 2007(1):059 608, doi:10.1155/2007/59608. URL http: / / jwcn.eurasipjournals.com/content/2007/1/059608.

42. Liu L, Chen R, Geirhofer S, Sayana K, Shi Z, Zhou Y. Downlink MIMO in LTE-advanced: SU-MIMO vs. MUMIMO. IEEE Communications Magazine Feb 2012; 50(2):140-147, doi:10.1109/MCOM.2012.6146493.

43. Lee D, Seo H, Clerckx B, Hardouin E, Mazzarese D, Nagata S, Sayana K. Coordinated multipoint transmission and reception in LTE-advanced: deployment scenarios and operational challenges. IEEE Communications Magazine Feb 2012; 50(2):148-155, doi:10.1109/MCOM.2012.6146494.

44. Angelis CT, Louvros S. Performance analysis and optimization of downlink multi-user MIMO LTE for satellite communications. Personal Satellite Services, Lecture Notes of the Institute for Computer Sciences, Social Informatics and Telecommunications Engineering, vol. 52, Pillai P, Shorey R, Ferro E (eds.). Springer Berlin Heidelberg, 2013; 69-80, doi:10.1007/978-3-642-36787-8_9. URL http://dx.doi .org/10.1007/ $978-3-642-36787-8 \_9$.

45. Larsson E, Edfors O, Tufvesson F, Marzetta T. Massive MIMO for next generation wireless systems. IEEE Communications Magazine Feb 2014; 52(2):186-195, doi:10.1109/MCOM.2014.6736761.

46. Arnau J, Mosquera C. Dissection of multibeam satellite communications with a large-scale antenna system toolbox. Proceedings of 20th European Wireless Conference, Barcelona, Spain, 2014. 